\title{
The Analytic Classification of Plane Branches
}

\author{
Abramo Hefez뇨 and Marcelo E. Hernandes 2
}

\begin{abstract}
In this paper we give a solution to the open classical problem of analytic classification of plane branches.

AMS Subject Classification (2000). Primary: 14H20. Secondary: 14Q05, 14Q20, 32S10

Keywords: plane curve singularities, analytic classification
\end{abstract}

\section{INTRODUCTION}

The aim of this work is to present a solution to the problem of effective analytic classification of plane branches.

Oscar Zariski, in a course taught at the École Polytechnique [11], in 1973, inspired by the work of S. Ebey [4], exposed his research on the problem of analytic classification of plane branches belonging to a given equisingularity class. A great amount of work is dedicated there to the analysis of particular examples, showing the need of more effective methods to solve the problem.

To this respect, in the introduction of [11, Zariski wrote:

Le problème de la description complète de l'éspace des modules $M$ d'une classe d'équisingularité donneè est entièrement ouvert et les quelques exemples du chapitre $V$ montrent que $M$ a une structure trop complexe pour espérer répondre totalement à la question.

La question, pourtant plus restrictive, de la détermination de la dimension de la "composante générique" de $M$ n'est pas résolue.

The first non-trivial result in this direction was given by C. Delorme in [3], where he answered the above second question in a very particular case, describing the generic component of the moduli space for plane branches with one Puiseux pair and computing its dimension.

In this paper, we show how one can break the complexity of the moduli space by stratifying the given equisingularity class by means of a good numerical invariant that separates branches into finitely many types, such that analytic equivalence is each stratum is manageable.

This is accomplished by considering the sets $\Lambda$ of values of Kähler differentials on branches as finer numerical invariants than the semigroup of values $\Gamma$ which characterizes the equisingularity class. If one stratifies with

\footnotetext{
${ }^{1}$ partially supported by CNPq, PRONEX and PROCAD/CAPES.

${ }^{2}$ partially supported by Fundação Araucária and PROCAD/CAPES.
} 
such sets $\Lambda$ the open set of the affine space representing the parameter space of an equisingularity class, corresponding to Puiseux parametrizations with fixed Puiseux pairs, and pick a set of special representatives in a specified normal form for each stratum, then the group action that represents analytic equivalence becomes rather trivial, allowing us to solve in general the above problems posed by Zariski.

Our setup is similar to that of [11, adding to it two techniques with computational flavor. The first one is the use of a $S A G B I$ algorithm, due to L. Robbiano and M. Sweedler [10], that we adapted to our situation in [7] and [9], to describe privileged bases of the local rings of plane branches as well of the modules of Kähler differentials of these local rings, allowing us to compute the set of invariants $\Lambda$. The second technique is the algorithm of Complete Transversal due to J. W. Bruce, N. P. Kirk and A. A. du Plessis [2] that allows to determine all normal forms of map-germs under one of Mather's group action, but doesn't allow to predict a priori what will be the outgoing result. The strength of our method stems in the conjugation of these two tools that allows, via the existence of some differentials, to control each step of the Complete Transversal algorithm, giving explicitly all possible normal forms and conditions for the analytic equivalence of germs in normal forms.

All the results we obtain are effective, in the sense that there is an efficient algorithm that puts any plane branch into its normal form and it is easy to recognize whether two plane branches under normal form are equivalent or not. The whole process has been implemented 3 .

\section{Preliminaries}

Our ground field is the field $\mathbb{C}$ of complex numbers. Since all power series we will work with are finitely determined, with respect to the equivalence relations we will consider, the results in this work are valid in the formal context and in the analytic context, as well. In order to have a geometric interpretation of our objects, we will adopt the analytic point of view. The reader who desires to find all the known results quoted in this section is invited to consult [6], where they are gathered with their proofs.

We denote by $\mathcal{O}_{2}$ the ring $\mathbb{C}\{X, Y\}$ of convergent power series in two variables with coefficients in $\mathbb{C}$. Let $f$ be in the maximal ideal $\mathcal{M}_{2}$ of $\mathcal{O}_{2}$ and irreducible. Then the class of $f$ in $\mathcal{O}_{2}$, modulo associates, is called a plane branch and denoted by $(f)$. We will identify $(f)$ with the germ of analytic plane curve at the origin $\left\{(x, y) \in\left(\mathbb{C}^{2}, 0\right) ; f(x, y)=0\right\}$.

We say that two plane branches $\left(f_{1}\right)$ and $\left(f_{2}\right)$ are equisingular, writing $\left(f_{1}\right) \equiv\left(f_{2}\right)$ if and only if $\left(f_{1}\right)$ and $\left(f_{2}\right)$ are topologically equivalent as complex immersed germs of curves in $\left(\mathbb{C}^{2}, 0\right)$; that is, when there exists a homeomorphism $\Phi: U \rightarrow U^{\prime}$, where $U$ and $U^{\prime}$ are neighborhoods of

\footnotetext{
${ }^{3}$ cf. www.dma.uem.br/ hernandes/publications.html
} 
the origin in $\mathbb{C}^{2}$ such that $f_{1}$ (resp. $f_{2}$ ) is convergent in $U$ (resp. $U^{\prime}$ ) and $\Phi\left(\left(f_{1}\right) \cap U\right)=\left(f_{2}\right) \cap U^{\prime}$. The set of all plane branches which are equisingular to each other will be called an equisingularity class.

When the above transformation $\Phi$ is an analytic isomorphism, we say that $\left(f_{1}\right)$ and $\left(f_{2}\right)$ are analytically equivalent, or shortly equivalent, writing in this case $\left(f_{1}\right) \sim\left(f_{2}\right)$. So, two branches $\left(f_{1}\right)$ and $\left(f_{2}\right)$ are equivalent if, and only if, there are an automorphism $\Phi^{*}$ and a unit $u$, both of $\mathcal{O}_{2}$, such that $\Phi^{*}\left(f_{1}\right)=u f_{2}$.

If we denote by $\mathcal{O}_{f}$ the quotient ring $\mathcal{O}_{2} /\langle f\rangle$, where $\langle f\rangle$ represents the ideal generated by $f$, then one has $\left(f_{1}\right) \sim\left(f_{2}\right)$ if, and only if, $\mathcal{O}_{f_{1}} \simeq \mathcal{O}_{f_{2}}$, as $\mathbb{C}$-algebras.

Our main concern in this work is to perform the analytic classification of plane branches within a given equisingularity class.

Since the integral closure of $\mathcal{O}_{f}$ in its field of fractions is a complete discrete valuation ring, hence isomorphic to $\mathcal{O}_{1}=\mathbb{C}\{t\}$, any plane branch $f$ has a parametrization $\varphi(t)=(x(t), y(t))$ with $x(t)$ and $y(t)$ in $\mathcal{M}_{1}$, the maximal ideal of $\mathcal{O}_{1}$, not both identically zero, such that $f(x(t), y(t))=$ 0 . Conversely, any non-zero mapping $\varphi:(\mathbb{C}, 0) \rightarrow\left(\mathbb{C}^{2}, 0\right)$, also called a parametrization, determines a plane branch. We will call a parametrization primitive if it cannot be reparametrized by a power of a new variable.

It is a well known fact, already used in [11] (see also Lemma 2.2 in [1]) that, given two plane branches $\left(f_{1}\right)$ and $\left(f_{2}\right)$, parametrized, respectively, by $\varphi_{1}$ and $\varphi_{2}$, then $\left(f_{1}\right) \sim\left(f_{2}\right)$ if, and only if, $\varphi_{1}$ and $\varphi_{2}$ are $\mathcal{A}$-equivalent, writing $\varphi_{1} \sim_{\mathcal{A}} \varphi_{2}$, where $\mathcal{A}$-equivalence means that there exist germs of analytic isomorphisms $\sigma$ and $\rho$ of $\left(\mathbb{C}^{2}, 0\right)$ and $(\mathbb{C}, 0)$, respectively, such that $\varphi_{2}=\sigma \circ \varphi_{1} \circ \rho^{-1}$.

So, the analytic classification of plane branches reduces to the $\mathcal{A}$-classification of parametrizations, which we are going to undertake in this paper.

Any plane branch is known (cf. [11]) to be equivalent to a branch with a (primitive) Puiseux parametrization:

$$
\varphi(t)=(x(t), y(t))=\left(t^{\beta_{0}}, \sum_{i \geq \beta_{1}} a_{i} t^{i}\right)
$$

with $a_{i} \in \mathbb{C}, a_{\beta_{1}}=1, \beta_{0}<\beta_{1}, \beta_{0} \not \beta_{1}$ and $f \circ \varphi(t)=0$.

The characteristic exponents of $\varphi$ are the integers $\beta_{0}, \beta_{1}, \ldots$, where for $i \geq 1$,

$$
\beta_{i}=\min \left\{j ; a_{j} \neq 0 \text { and } \operatorname{GCD}\left(\beta_{0}, \ldots, \beta_{i-1}, j\right) \neq \operatorname{GCD}\left(\beta_{0}, \ldots, \beta_{i-1}\right)\right\} .
$$

The associated integers $n_{i}$ are $n_{0}=1$ and

$$
n_{i}=\frac{e_{i-1}}{e_{i}}
$$

where $e_{0}=\beta_{0}$ and $e_{i}=\operatorname{GCD}\left(\beta_{0}, \ldots, \beta_{i}\right)$. 
Since the parametrization is primitive, there is a positive integer $g$ such that $e_{g}=1$. This $g$ is called the genus of the branch. The Puiseux pairs of $\varphi$ are $\left(n_{i}, m_{i}\right), i=1, \ldots, g$, where $m_{i}=\frac{\beta_{i}}{e_{i}}$.

It is a classical result, going back to the thirties, due essentially to Brauner and Zariski, that the Puiseux pairs form a complete set of numerical invariants for the topological classification of plane branches; that is, if $f_{1}$ and $f_{2}$ have parametrizations as $(2.1)$, then $\left(f_{1}\right) \equiv\left(f_{2}\right)$ if, and only if, $\left(f_{1}\right)$ and $\left(f_{2}\right)$ have the same Puiseux pairs or, equivalently, the same characteristic exponents.

So, the Puiseux parametrizations as (2.1) with $a_{\beta_{i}} \neq 0$, for $i=2, \ldots, g$, and such that $a_{j}=0$, if $\beta_{i} \leq j<\beta_{i+1}$ and $e_{i} \not \chi j$, determine equisingular branches; and any plane branch in this equisingularity class is equivalent to one with such a Puiseux parametrization.

Any parametrization $\varphi$ induces a homomorphism

$$
\begin{aligned}
& \varphi^{*}: \mathcal{O}_{2} \rightarrow \mathcal{O}_{1} . \\
& h \mapsto h \circ \varphi(t)
\end{aligned}
$$

Assuming $\varphi$ primitive, we define the value $v_{\varphi}(h)$, for $h \in \mathcal{O}_{2}$, as being $\operatorname{ord}_{t}\left(\varphi^{*}(h)\right)$, the order in $t$ of the power series $\varphi^{*}(h)$, defining also $v_{\varphi}(0)=\infty$. The semigroup of the extended naturals $\Gamma_{\varphi}=v_{\varphi}\left(\mathcal{O}_{2}\right)$ will be called the semigroup of values of $\varphi$. This semigroup will be represented by $\Gamma_{\varphi}=\left\langle v_{0}, v_{1}, \ldots, v_{g}\right\rangle$, where $v_{0}<\cdots<v_{g}$ is its minimal set of generators. When $\varphi$ is a Puiseux parametrization, it is well known (cf. [11]) that there are relations among these $v_{i}$ 's and the $\beta_{i}$ 's, given by $\beta_{0}=v_{0}, \beta_{1}=v_{1}$, $\operatorname{GCD}\left(v_{0}, \ldots, v_{i}\right)=e_{i}$, and

$$
v_{i+1}=n_{i} v_{i}+\beta_{i+1}-\beta_{i} .
$$

Hence, the characteristic exponents of $\varphi$ and $\Gamma_{\varphi}$ determine each other.

The semigroup $\Gamma_{\varphi}$ has a conductor; that is, there is a natural number $c$ such that $c-1 \notin \Gamma_{\varphi}$, and $l \in \Gamma_{\varphi}$, for all $l \geq c$. This means that the set $\mathbb{N} \backslash \Gamma_{\varphi}$, whose elements are called the gaps of $\Gamma_{\varphi}$, is finite.

So, in order to preserve the Puiseux form given in (2.1), under the $\mathcal{A}$ action, the analytic isomorphisms $\sigma$ and $\rho$ must be of a very special type, as described below.

If $\varphi(t)=(x(t), y(t))$ and $\varphi_{1}\left(t_{1}\right)=\left(x_{1}\left(t_{1}\right), y_{1}\left(t_{1}\right)\right)$ are two parametrizations as in (2.1), then in order to have $\varphi_{1}\left(t_{1}\right)=\sigma \circ \varphi \circ \rho^{-1}\left(t_{1}\right)$, it is necessary and sufficient that

$$
\begin{aligned}
& \sigma(X, Y)=\left(r^{v_{0}} X+p, r^{v_{1}} Y+q\right), \\
& t_{1}=\rho(t)=r t \sqrt[v_{0}]{1+\frac{\varphi^{*}(p)}{r^{v_{0}} t^{v_{0}}}},
\end{aligned}
$$

where $r \in \mathbb{C}^{*}$ and $p, q \in \mathcal{O}_{2}$, with $v_{\varphi}(p)>v_{0}$ and $v_{\varphi}(q)>v_{1}$. 
The $\mathcal{A}$-action induced on parametrizations of the form (2.1) is then given by $\left(t^{v_{0}}, y(t)\right) \sim_{\mathcal{A}}\left(t_{1}^{v_{0}}, y_{1}\left(t_{1}\right)\right)$ if, and only if,

$$
y_{1}\left(t_{1}\right)=r^{v_{1}} y\left(\rho^{-1}\left(t_{1}\right)\right)+q\left(\rho^{-1}\left(t_{1}\right)^{v_{0}}, y\left(\rho^{-1}\left(t_{1}\right)\right)\right) .
$$

Ebey and Zariski (cf. [4] and [1]) gave some elimination criteria (EC) of parameters of $y(t)$ in a given parametrization $\left(t^{v_{0}}, y(t)\right)$ as in $(2.1)$, by means of the $\mathcal{A}$-equivalence.

Let $\varphi=\left(t^{v_{0}}, t^{v_{1}}+\sum_{i>v_{1}} a_{i} t^{i}\right)$ be a Puiseux parametrization, and let $j>v_{1}$ be an integer. If one of the following conditions holds,

EC1) $j \in \Gamma_{\varphi}$, or

EC2) $j+v_{0}-v_{1} \in \Gamma_{\varphi}$,

then $\varphi$ is $\mathcal{A}$-equivalent to a parametrization $\left(t^{v_{0}}, t^{v_{1}}+\sum_{i>v_{1}} a_{i}^{\prime} t^{i}\right)$, with $a_{i}^{\prime}=$ $a_{i}$, when $i<j$, and $a_{j}^{\prime}=0$.

It then follows that any parametrization $\varphi=\left(t^{v_{0}}, \sum_{v_{1} \leq i} a_{i} t^{i}\right)$ is $\mathcal{A}$-equivalent to the parametrization

$$
\left(t^{v_{0}}, \sum_{v_{1} \leq i<c} a_{i} t^{i}\right)
$$

where $c$ is the conductor of $\Gamma_{\varphi}$.

Let $\Sigma_{\Gamma}$ denote the set of all parametrizations of the above form, such that $\Gamma_{\varphi}$ is equal to a given $\Gamma$. This set can be identified with an open set (the complement of the union of the hyperplanes $a_{\beta_{i}}=0, i=2, \ldots, g$ ) of an affine space, whose points are the ordered sets of the coefficients of $y(t)$ which are not necessarily zero, excluding $a_{\beta_{1}}$, which is taken to be 1 . So, now, we are reduced to classify, modulo $\mathcal{A}$-equivalence, the parametrizations in the set $\Sigma_{\Gamma}$, in order to classify analytically plane branches.

Zariski noticed in [11] that, in order to get more Elimination Criteria then the above ones, it was necessary to consider the module of Kähler differentials over the local ring of the branch, introducing new numerical analytic invariants.

Let us denote by

$$
\Omega_{2}=\left\{h d X+g d Y ; \quad g, h \in \mathcal{O}_{2}\right\},
$$

the $\mathcal{O}_{2}$-free module of germs of differentials at $\left(\mathbb{C}^{2}, 0\right)$, and by

$$
\Omega_{1}=\left\{\xi d t ; \quad \xi \in \mathcal{O}_{1}\right\},
$$

the $\mathcal{O}_{1}$-free module of germs of differentials at $(\mathbb{C}, 0)$.

A parametrization $\varphi:(\mathbb{C}, 0) \rightarrow\left(\mathbb{C}^{2}, 0\right), t \mapsto(x(t), y(t))$, induces a natural $\mathcal{O}_{2}$-modules homomorphism (thought as an extension of the map $\varphi^{*}: \mathcal{O}_{2} \rightarrow$ $\left.\mathcal{O}_{1}\right)$ :

$$
\begin{aligned}
& \varphi^{*}: \quad \Omega_{2} \quad \longrightarrow \quad \Omega_{1} \text {. } \\
& h d X+g d Y \quad \mapsto \quad\left(\varphi^{*}(h) x^{\prime}(t)+\varphi^{*}(g) y^{\prime}(t)\right) d t
\end{aligned}
$$

The image of $\Omega_{2}$ in $\Omega_{1}$ under $\varphi^{*}$ is isomorphic to the quotient of the module of Kähler differentials over the local ring $\left(\varphi^{*}\left(\mathcal{O}_{2}\right)\right)$ of the branch determined by $\varphi$, by its torsion submodule (cf. [7] or [9]). 
A primitive parametrization $\varphi$ also induces a valuation $v_{\varphi}$ on $\Omega_{2}$, defined by

$$
v_{\varphi}(h d X+g d Y)=\operatorname{ord}_{t}\left(\varphi^{*}(h) x^{\prime}(t)+\varphi^{*}(g) y^{\prime}(t)\right)+1 .
$$

We now define

$$
\Lambda_{\varphi}=v_{\varphi}\left(\Omega_{2}\right) .
$$

The set $\Lambda_{\varphi}$ is $\mathcal{A}$-invariant, as we will show later, and will play a key role in our solution of the classification problem.

Since for all $h \in \mathcal{M}_{2}$ we have that $v_{\varphi}(d h)=v_{\varphi}(h)$, it follows that $\Gamma_{\varphi} \backslash\{0\} \subset$ $\Lambda_{\varphi}$; and because $\Gamma_{\varphi}$ has a conductor, we have that the set $\Lambda_{\varphi} \backslash \Gamma_{\varphi}$, as a subset of the set of gaps of $\Gamma_{\varphi}$, is finite.

Zariski in [12] has shown that $\Lambda_{\varphi} \backslash \Gamma_{\varphi}=\emptyset$ if, and only if, $\varphi$ is $\mathcal{A}$-equivalent to the parametrization $\left(t^{v_{0}}, t^{v_{1}}\right)$, where $\operatorname{GCD}\left(v_{0}, v_{1}\right)=1$.

Since $\Lambda_{\varphi}$ and $\Gamma_{\varphi}$ are $\mathcal{A}$-invariant, it follows that, if $\Lambda_{\varphi} \backslash \Gamma_{\varphi} \neq \emptyset$, then

$$
\lambda=\min \left(\Lambda_{\varphi} \backslash \Gamma_{\varphi}\right)-v_{0},
$$

is an invariant under the $\mathcal{A}$-action, called the Zariski invariant of $\varphi$. It is known (see [11]) that such a $\varphi$ is $\mathcal{A}$-equivalent to a Puiseux parametrization of the form

$$
\varphi=\left(t^{v_{0}}, t^{v_{1}}+t^{\lambda}+\sum_{\lambda<i<c} a_{i} t^{i}\right)
$$

and, in this case,

$$
\lambda=v_{\varphi}\left(v_{0} X d Y-v_{1} Y d X\right)-v_{0} .
$$

Related to the invariant $\lambda$, Zariski in [11] proved the following extra elimination criterion:

EC3) If $\varphi$ is as in (2.4) and $j-\lambda$ is in the semigroup generated by $v_{0}$ and $v_{1}$, then $\varphi$ is $\mathcal{A}$-equivalent to a parametrization $\left(t^{v_{0}}, t^{v_{1}}+t^{\lambda}+\sum_{\lambda<i<c} a_{i}^{\prime} t^{i}\right)$, with $a_{i}^{\prime}=a_{i}$, when $i<j$, and $a_{j}^{\prime}=0$.

The above criterion doesn't work for all the equisingularity class, but depends upon the $\mathcal{A}$-equivalence class of the parametrization $\varphi$.

In the next theorem, our central result in this work, we will determine all possible such elimination criteria, which will lead us to what we call the normal forms for the Puiseux parametrizations.

Theorem 2.1 (The Normal Forms Theorem). Let $\varphi \in \Sigma_{\Gamma}$ be a Puiseux parametrization of a plane branch with semigroup of values $\Gamma=\left\langle v_{0}, v_{1}, \ldots, v_{g}\right\rangle$. Then, either $\varphi$ is $\mathcal{A}$-equivalent to the monomial parametrization $\left(t^{v_{0}}, t^{v_{1}}\right)$, or it is $\mathcal{A}$-equivalent to a parametrization

$$
\left(t^{v_{0}}, t^{v_{1}}+t^{\lambda}+\sum_{\substack{i>\lambda \\ i \notin \Lambda-v_{0}}} a_{i} t^{i}\right),
$$

where $\lambda$ is its Zariski invariant and $\Lambda=\Lambda_{\varphi}$ is the set of orders of differentials of the branch. Moreover, if $\varphi$ and $\varphi^{\prime}$ (with coefficients $a_{i}^{\prime}$ instead of $a_{i}$ ) are parametrizations as in (2.5), representing two plane branches with same 
semigroup of values and same set of values of differentials, then $\varphi \sim_{\mathcal{A}} \varphi^{\prime}$ if and only if there is $r \in \mathbb{C}^{*}$ such that $r^{\lambda-v_{1}}=1$ and $a_{i}=r^{i-v_{1}} a_{i}^{\prime}$, for all $i$.

Remark that the $\mathcal{A}$-normal form in (2.5) is completely determined by the semigroup $\Gamma$ and the set $\Lambda$. So, once $\Gamma$ is fixed, the number of $\mathcal{A}$-normal forms is finite, corresponding to all possible sets $\Lambda$ in the equisingularity class determined by $\Gamma$, which may be computed by the algorithm presented in 9 .

The above theorem gives the ultimate elimination criterion EC that contains all the known criteria EC1, EC2 and EC3:

EC) If $\varphi$ is as in (2.4) and $j+v_{0} \in \Lambda_{\varphi}, j>\lambda$, then $\varphi$ is $\mathcal{A}$-equivalent to a parametrization $\left(t^{v_{0}}, t^{v_{1}}+t^{\lambda}+\sum_{\lambda<i<c} a_{i}^{\prime} t^{i}\right)$, with $a_{i}^{\prime}=a_{i}$, when $i<j$, and $a_{j}^{\prime}=0$.

The rest of the paper is devoted to prove Theorem 2.1.

\section{Orbits And their TAngent Spaces}

We will assume the reader familiar with the language of singularity theory. We will denote by $j^{k}(h)$ the $k$-jet of an element $h$.

Let $\operatorname{Aut}\left(\mathbb{C}^{n}, 0\right)$ denote the group of germs of analytic automorphisms of $\left(\mathbb{C}^{n}, 0\right)$, and let $\operatorname{Aut}_{1}\left(\mathbb{C}^{n}, 0\right)$ the subgroup of elements $A \in \operatorname{Aut}\left(\mathbb{C}^{n}, 0\right)$ such that $j^{1}(A)=$ Id.

We also denote by $\widetilde{\operatorname{Aut}}\left(\mathbb{C}^{2}, 0\right)$ the subgroup of elements $A$ of $\operatorname{Aut}\left(\mathbb{C}^{2}, 0\right)$ such that $j^{1}(A)=(X+\beta Y, Y)$, with $\beta \in \mathbb{C}$.

We say that the Puiseux parametrizations $\varphi_{1}$ and $\varphi_{2}$ are $\mathcal{A}_{1}$-equivalent (resp. $\widetilde{\mathcal{A}}$-equivalent) if $\varphi_{2}=\sigma \circ \varphi_{1} \circ \rho^{-1}$ with $\sigma \in \operatorname{Aut}_{1}\left(\mathbb{C}^{2}, 0\right)$ (resp. $\sigma \in$ $\left.\widetilde{\operatorname{Aut}}\left(\mathbb{C}^{2}, 0\right)\right)$ and $\rho \in \operatorname{Aut}_{1}(\mathbb{C}, 0)$.

We say that $\varphi_{1}$ and $\varphi_{2}$ in $\Sigma_{\Gamma}$ are homothetic, or $\mathcal{H}$-equivalent if $\varphi_{2}=$ $\sigma \circ \varphi_{1} \circ \rho^{-1}$, with $\rho(t)=\alpha t$ and $\sigma(X, Y)=\left(\alpha^{v_{0}} X, \alpha^{v_{1}} Y\right)$, for some $\alpha \in \mathbb{C}^{*}$.

So, the $\mathcal{A}$-action on the space of Puiseux series representing an equisingularity class may be obtained by the $\widetilde{\mathcal{A}}$-action followed by the $\mathcal{H}$-action.

If $\mathcal{G}$ represents one of the actions $\mathcal{A}, \mathcal{A}_{1}$ or $\widetilde{\mathcal{A}}$, then $\mathcal{G}^{k}$ will represent the Lie group action of $k$-jets of corresponding automorphisms on the space $\Sigma_{\Gamma}^{k}$ of $k$-jets of elements of $\Sigma_{\Gamma}$.

Let us recall (a special case) of the Complete Transversal Theorem of [2], adapted to our use:

The Complete Transversal Theorem. Let $G$ be a Lie group acting smoothly on an open set $U$ of an affine space $A$ with underlying vector space $V$, and let $W$ be a subspace of $V$ such that $\forall g \in G, \forall v \in U$ and $\forall w \in W$ with $v+w \in U$ and $g \cdot v+w \in U$, one has

$$
g \cdot(v+w)=g \cdot v+w .
$$

If $W \subset T_{v}(G \cdot v)$, with $v \in U$, and $T_{v}(G \cdot v)$ is the tangent space at $v$ to the orbit $G \cdot v$, then for every $w \in W$ such that $v+w \in U$, one has

$$
G(v+w)=G \cdot v \text {. }
$$


Although we are mainly interested in the $\mathcal{A}$-equivalence, we will start analyzing the unipotent $\mathcal{A}_{1}$-action, passing to the $\widetilde{\mathcal{A}}$-action and, finally, applying homotheties, to get to the $\mathcal{A}$-equivalence.

Let $U$ be the open set $\Sigma_{\Gamma}^{k}$ of the appropriate affine space and let $G=\mathcal{A}_{1}^{k}$. Then the initial hypothesis at the beginning of the Complete Transversal Theorem is fulfilled for $W=\left\{\left(0, b t^{k}\right) ; b \in \mathbb{C}\right\}$.

The tangent spaces to the orbits $\mathcal{A}_{1}^{k} \cdot \varphi$ and $\widetilde{\mathcal{A}}^{k} \cdot \varphi$ at an element $\varphi=$ $(x(t), y(t)) \in \Sigma_{\Gamma}^{k}$ are given by:

$$
T_{\varphi}\left(\mathcal{A}_{1}^{k} \cdot \varphi\right)=\left\{j^{k}\left(\left(x^{\prime}(t), y^{\prime}(t)\right) \epsilon+\left(\varphi^{*}(g), \varphi^{*}(h)\right) ; \epsilon \in \mathcal{M}_{1}^{2}, g, h \in \mathcal{M}_{2}^{2}\right\}\right.
$$

and

$$
\begin{array}{r}
T_{\varphi}\left(\widetilde{\mathcal{A}}^{k} \cdot \varphi\right)=\left\{j ^ { k } \left(\left(x^{\prime}(t), y^{\prime}(t)\right) \epsilon+\left(\varphi^{*}(g), \varphi^{*}(h)\right) ;\right.\right. \\
\epsilon \in \mathcal{M}_{1}^{2}, h \in \mathcal{M}_{2}^{2}, \\
\left.g \in\left\langle X^{2}, Y\right\rangle\right\} .
\end{array}
$$

The proof of (3.1) may be found, for example, in [5], while the proof of (3.2) may be obtained in a similar way.

We will show how, by using the Complete Transversal Theorem, one obtains all normal forms of Puiseux parametrizations, with respect to the $\mathcal{A}_{1}$ equivalence, by eliminating terms in the expansion of $y(t)$, finding more elimination criteria than the general ones of Ebey and Zariski, adapted to a specific branch. The idea is to verify at each step if the $k$-jet of the parametrization is $\mathcal{A}_{1}^{k}$-equivalent to its $(k-1)$-jet, which implies that the term of degree $k$ in $y(t)$ can be eliminated under the $\mathcal{A}_{1}$-action. For this, according to the Complete Transversal Theorem, it is enough to verify if the vector $\left(0, b t^{k}\right)$ belongs to the tangent space to the $\mathcal{A}_{1}^{k}$-orbit of the $k$ jet of the parametrization, and this fact may be expressed in terms of the existence of differentials in $\left(\mathbb{C}^{2}, 0\right)$ of certain order with respect to the valuation determined by the parametrization, as we will see soon. The procedure will stop after finitely many steps since all terms in $y(t)$ of order greater or equal to the conductor $c$ of the semigroup of values of the branch are eliminable. Next, we will find the normal forms under the $\widetilde{\mathcal{A}}$-action by analyzing separately some few remaining cases. Finally, the normal forms under the $\mathcal{A}$-action are obtained applying homotheties.

In order to apply this procedure, we will need to describe more explicitly the tangent spaces to orbits in $\Sigma_{\Gamma}^{k}$.

Lemma 3.1. Let $k>v_{1}$ and $\varphi \in \Sigma_{\Gamma}^{k}$. For $b \neq 0$, we have that the vector $\left(0, b t^{k}\right)$ belongs to $T_{\varphi}\left(\mathcal{A}_{1}^{k} \cdot \varphi\right)$ (resp. to $T_{\varphi}\left(\widetilde{\mathcal{A}}^{k} \cdot \varphi\right)$ ), if and only if there exist $g, h \in \mathcal{M}_{2}^{2}$ (resp. $g \in\left\langle X^{2}, Y\right\rangle, h \in \mathcal{M}_{2}^{2}$ ) such that

$$
k+v_{0}-1=\operatorname{ord}_{t}\left(\varphi^{*}(h) x^{\prime}(t)-\varphi^{*}(g) y^{\prime}(t)\right) .
$$

Proof: We prove the result for $T_{\varphi}\left(\mathcal{A}_{1}^{k} \cdot \varphi\right)$, since the other situation is similar. 
In order to have $\left(0, b t^{k}\right) \in T_{\varphi}\left(\mathcal{A}_{1}^{k} \cdot \varphi\right)$ it is necessary and sufficient to be able to solve the system:

$$
\begin{cases}x^{\prime}(t) \cdot \epsilon+\varphi^{*}(g)=0 & \bmod t^{k+1} \\ y^{\prime}(t) \cdot \epsilon+\varphi^{*}(h)=b t^{k} & \bmod t^{k+1}\end{cases}
$$

That is, $\epsilon=-\frac{\varphi^{*}(g)}{x^{\prime}(t)} \bmod t^{k+1}$. Notice that $\epsilon \in \mathcal{M}_{1}^{2}$, since $g \in \mathcal{M}_{2}^{2}$.

In this way we get the equation

$$
b t^{k}=\frac{\varphi^{*}(h) x^{\prime}(t)-\varphi^{*}(g) y^{\prime}(t)}{x^{\prime}(t)} \bmod t^{k+1} .
$$

So, $\left(0, b t^{k}\right) \in T_{\varphi}\left(\mathcal{A}_{1}^{k} \cdot \varphi\right)$ if, and only if, there exist $g, h \in \mathcal{M}_{2}^{2}$ satisfying (3.3).

For $i \in \mathbb{N}$, we define

$$
\Omega_{2}^{(i)}=\left\{h d X+g d Y \in \Omega_{2} ; \quad g, h \in \mathcal{M}_{2}^{i}\right\},
$$

where we put $\mathcal{M}_{2}^{0}=\mathcal{O}_{2}$. So, $\Omega_{2}^{(0)}=\Omega_{2}$.

Given a primitive parametrization $\varphi$, we also define

$$
\Lambda_{\varphi}^{i}=v_{\varphi}\left(\Omega_{2}^{(i)}\right)
$$

Notice that $\Lambda_{\varphi}^{0}=\Lambda_{\varphi}$. These sets are invariant under $\mathcal{A}$-equivalence, as we show below.

Proposition 3.2. If $\varphi$ and $\varphi_{1}$ are $\mathcal{A}$-equivalent primitive parametrizations, then, for all $i \in \mathbb{N}$, we have $\Lambda_{\varphi}^{i}=\Lambda_{\varphi_{1}}^{i}$.

Proof: The commutative diagram,

$$
\begin{array}{lll}
\mathbb{C}, 0 & \stackrel{\varphi}{\longrightarrow} & \mathbb{C}^{2}, 0 \\
\downarrow \rho & & \downarrow \sigma \\
\mathbb{C}, 0 & \stackrel{\varphi_{1}}{\longrightarrow} & \mathbb{C}^{2}, 0
\end{array}
$$

with isomorphisms $\sigma$ an $\rho$ induces the following two diagrams:

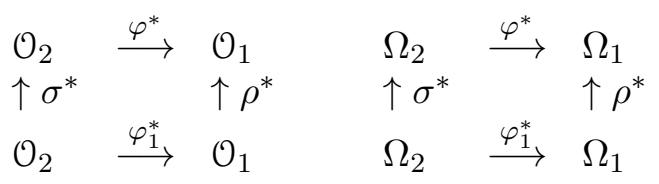

with isomorphisms $\sigma^{*}$ and $\rho^{*}$. Now the result follows by functoriality, observing that $\sigma^{*}\left(\mathcal{M}_{2}^{i}\right)=\mathcal{M}_{2}^{i}$.

If we define

$$
\Omega_{2}^{\prime}=\left\{h d X+g d Y \in \Omega_{2} ; \quad g \in\left\langle X^{2}, Y\right\rangle, h \in \mathcal{M}_{2}^{2}\right\},
$$

and $\Lambda_{\varphi}^{\prime}=v_{\varphi}\left(\Omega_{2}^{\prime}\right)$, then Lemma 3.1 may be rephrased as follows: 
Proposition 3.3. Let $k>v_{1}$ and $\varphi \in \Sigma_{\Gamma}^{k}$. For $b \neq 0$, we have that $\left(0, b t^{k}\right)$ belongs to $T_{\varphi}\left(\mathcal{A}_{1}^{k} \cdot \varphi\right)$ (resp. to $\left.T_{\varphi}\left(\widetilde{\mathcal{A}}^{k} \cdot \varphi\right)\right)$ if, and only if,

$$
k+v_{0} \in \Lambda_{\varphi}^{2} \quad\left(\text { resp. } k+v_{0} \in \Lambda_{\varphi}^{\prime}\right)
$$

\section{Normal $\mathcal{A}_{1}$-Forms}

In this section we will find the normal forms of Puiseux parametrizations in an equisingularity class with given semigroup of values $\Gamma$, under $\mathcal{A}_{1^{-}}$ equivalence. We begin with a proposition that will give us the recursion step.

Proposition 4.1. Let $\varphi=\left(t^{v_{0}}, t^{v_{1}}+\sum_{v_{1}<i<c} a_{i} t^{i}\right) \in \Sigma_{\Gamma}$ and let $k$ be an integer such that $k+v_{0} \in \Lambda_{\varphi}^{2}$. Then there exists $\varphi_{1} \in \Sigma_{\Gamma}$ such that $\varphi_{1} \sim_{\mathcal{A}_{1}} \varphi$ and

$$
j^{k}\left(\varphi_{1}\right)=j^{k-1}\left(\varphi_{1}\right)=j^{k-1}(\varphi) .
$$

Proof: From Proposition 3.3, we have that the vector $\left(0,-a_{k} t^{k}\right)$ belongs to $T_{j^{k}(\varphi)}\left(\mathcal{A}_{1}^{k} \cdot j^{k}(\varphi)\right)$, and therefore by the Complete Transversal Theorem it follows that $j^{k}(\varphi) \sim_{\mathcal{A}_{1}^{k}} j^{k-1}(\varphi)$. Hence, there are appropriate germs of analytic isomorphisms $\sigma$ and $\rho$ such that $\sigma \circ j^{k}(\varphi) \circ \rho^{-1}=j^{k-1}(\varphi)$. So, $j^{k}\left(\sigma \circ \varphi \circ \rho^{-1}\right)=j^{k-1}(\varphi)$. Now the result follows putting $\varphi_{1}=\sigma \circ \varphi \circ \rho^{-1}$

The following result will be important in the sequel.

Proposition 4.2. Let $\varphi=\left(t^{v_{0}}, t^{v_{1}}+t^{\lambda}+\cdots\right)$ be a Puiseux parametrization with $\Gamma_{\varphi}=\left\langle v_{0}, v_{1}, \ldots, v_{g}\right\rangle$. If $S=\left\{v_{0}, 2 v_{0}, v_{1}, v_{0}+v_{1}, 2 v_{1}, v_{0}+\lambda\right\}$, then one has

$$
S \subseteq \Lambda_{\varphi} \backslash \Lambda_{\varphi}^{2} \subseteq S \cup\left\{v_{1}+\lambda\right\}
$$

with equality on the top if, and only if, $n_{1}=2$ and $g \geq 2$.

Proof: We have that $n \in \Lambda_{\varphi} \backslash \Lambda_{\varphi}^{2}$ if, and only if, $n=v_{\varphi}(\omega)$, where $\omega=h d X+g d Y$, with $h \notin \mathcal{M}_{2}^{2}$ or $g \notin \mathcal{M}_{2}^{2}$.

We have that $S \subseteq \Lambda_{\varphi} \backslash \Lambda_{\varphi}^{2}$, since $v_{\varphi}(d X)=v_{0}, v_{\varphi}(d Y)=v_{1}, v_{\varphi}(X d X)=$ $2 v_{0}, v_{\varphi}(X d Y)=v_{0}+v_{1}, v_{\varphi}(Y d Y)=2 v_{1}$, and $v_{\varphi}\left(v_{1} Y d X-v_{0} X d Y\right)=v_{0}+\lambda$.

Now, suppose that $v_{\varphi}(h d X+g d Y) \notin S$, where $h=\alpha X+\beta Y+h_{2}$ and $g=a X+b Y+g_{2}$, with $h_{2}, g_{2} \in \mathcal{M}_{2}^{2}$, and one of the numbers $a, b, \alpha$ or $\beta$ is not zero.

So, in this case, we must have $v_{\varphi}(h d X+g d Y)>v_{\varphi}(h d X)=v_{\varphi}(g d Y)$. This implies that

$$
v_{\varphi}(h)+v_{0}=v_{\varphi}(g)+v_{1},
$$

with $v_{\varphi}(h)=v_{0}$ or $v_{\varphi}(h)=v_{1}$ or $v_{\varphi}(g)=v_{0}$ or $v_{\varphi}(g)=v_{1}$.

If $v_{\varphi}(h)=v_{0}$, then we would have $2 v_{0}=v_{\varphi}(g)+v_{1}$, which is not possible. Hence $\alpha=0$.

If $v_{\varphi}(g)=v_{0}$, then $v_{\varphi}(h)+v_{0}=v_{1}+v_{0}$, hence $v_{\varphi}(h)=v_{1}$. 
If $v_{\varphi}(h)=v_{1}$, then $v_{1}+v_{0}=v_{\varphi}(g)+v_{1}$, so $v_{\varphi}(g)=v_{0}$. Hence, in this case, $v_{\varphi}(h d X+g d Y)=v_{0}+\lambda \in S$, which is to be excluded. Hence, $a=\beta=0$.

So, the only remaining possibility is that $v_{\varphi}(g)=v_{1}$, in which case, $b \neq 0$ and $a=\alpha=\beta=0$. So, we have $v_{\varphi}(h)+v_{0}=2 v_{1}$, hence $v_{1}<v_{\varphi}(h)<2 v_{1}$, which implies that $v_{\varphi}(h)=s v_{1}+r v_{0}$, with $s=0,1$. We have that $s=0$, because, otherwise, we would have $v_{1}+(r+1) v_{0}=2 v_{1}$, which would imply that $v_{0}$ divides $v_{1}$, a contradiction.

If the genus of $\varphi$ is 1 , then $v_{0}=2$, and in this case, because of EC1 we have that $\varphi$ is $\mathcal{A}$-equivalent to the parametrization $\left(t^{2}, t^{v_{1}}\right)$, hence not satisfying the hypothesis of the Proposition.

Therefore, $g \geq 2$, and $v_{\varphi}(h)=r v_{0}$. Therefore,

$$
(r+1) v_{0}=2 v_{1},
$$

which implies $n_{1}=2$. Also, $v_{\varphi}(h d X+g d Y)>2 v_{1}$, which in view of the expression of $\varphi$ and the above equality implies that $v_{\varphi}(h d X+g d Y)=v_{1}+\lambda$.

Conversely, if $g \geq 2$ and $n_{1}=2$, we have that

$$
v_{\varphi}\left(v_{1} X^{r} d X-v_{0} Y d Y\right)=v_{1}+\lambda
$$

where $(r+1) v_{0}=2 v_{1}$.

Now, we have the following result:

Proposition 4.3. Let $\varphi \in \Sigma_{\Gamma}$ and set $\Lambda=\Lambda_{\varphi}$. Suppose that $\Lambda \backslash \Gamma \neq$ $\emptyset$, and let $\lambda$ be the Zariski invariant of $\varphi$. Then $\varphi$ is $\mathcal{A}_{1}$-equivalent to a parametrization

$$
\left(t^{v_{0}}, t^{v_{1}}+t^{\lambda}+\sum_{\substack{i \notin \Lambda^{2}-v_{0} \\ i>\lambda}} a_{i} t^{i}\right) .
$$

Proof: First observe that since $\Lambda \backslash \Gamma \neq \emptyset$, it follows that $v_{0} \geq 3$, so, in this situation, any integer $l+v_{0}$, where $l$ is greater or equal than the conductor $c$ of $\Gamma$, belongs to $\Lambda$ but, by Proposition 4.2, it is not in $\Lambda \backslash \Lambda^{2}$, so it is in $\Lambda^{2}$. This shows that the set $\mathbb{N} \backslash\left(\Lambda^{2}-v_{0}\right)$ is finite (bounded by above by $\left.c-1\right)$.

Let $\lambda_{1}, \ldots, \lambda_{s}$ be the elements in $\Lambda^{2}-v_{0}$ in the interval $(\lambda, c)$. From Proposition 4.1, there exists a Puiseux parametrization $\varphi_{1}$ with $\varphi_{1} \sim_{\mathcal{A}_{1}} \varphi$ such that

$$
j^{\lambda_{1}}\left(\varphi_{1}\right)=j^{\lambda_{1}-1}\left(\varphi_{1}\right)=j^{\lambda_{1}-1}(\varphi) .
$$

Next, do the same with $\varphi_{1}$ instead of $\varphi$ and $\lambda_{2}$ instead of $\lambda_{1}$, observing, by Proposition 3.2, that $\Lambda_{\varphi_{1}}^{2}=\Lambda^{2}$; etc.

The next step will be to pass from the $\mathcal{A}_{1}$-equivalence to the $\widetilde{\mathcal{A}}$-equivalence. 


\section{Passage from the $\mathcal{A}_{1}$-EQuivalence to the $\widetilde{\mathcal{A}}$-EQuivalence}

To get the normal forms of Theorem 2.1, in view of the result of Proposition 4.3, it suffices to show that the terms in $y(t)$ of a Puiseux parametrization of order $k$ such that $k>\lambda$ and $k \in\left(\Lambda \backslash \Lambda^{2}\right)-v_{0}$, may be eliminated without changing the preceding terms.

Terms of order $k \in S-v_{0}$, where $S$ is as in Proposition 4.2, excepting $k=\lambda$, may be eliminated by EC2. The only remaining possibility are terms of order $v_{1}+\lambda-v_{0}$, when $g \geq 2$ and $n_{1}=2$ (cf. Proposition 4.2), which we will show below how to eliminate them without changing the preceding ones.

To do this, we will need to analyze more closely the $\widetilde{\mathcal{A}}$-action on Puiseux parametrizations.

Let $\varphi(t)=\left(t^{v_{0}}, y(t)\right)$, where $y(t)=\sum_{i} a_{i} t^{i}$, and let $\sigma$ and $\rho$ as in (2.2), but with $r=1, p=\beta Y+p_{1}$, where $\beta \in \mathbb{C}$ and $p_{1}, q \in \mathcal{M}_{2}^{2}$.

Now, considering the expression of $\rho$ in (2.2), raising both sides to the power $i$ and then applying the binomial expansion we get

$$
t_{1}^{i}=t^{i}\left[\sum_{j=0}^{\infty}\left(\begin{array}{c}
i / v_{0} \\
j
\end{array}\right)\left(\frac{p(t)}{t^{v_{0}}}\right)^{j}\right],
$$

where $p(t)=\varphi^{*}(p)$.

By using this in the expression $y(t)=\sum_{i} a_{i} t^{i}$, we get

$$
y\left(t_{1}\right)=y(t)+\sum_{i} a_{i} t^{i} \frac{i}{v_{0}} \frac{p(t)}{t^{v_{0}}}+A(t),
$$

where

$$
A(t)=\sum_{i} a_{i} t^{i} \sum_{j=2}^{\infty}\left(\begin{array}{c}
i / v_{0} \\
j
\end{array}\right)\left(\frac{p(t)}{t^{v_{0}}}\right)^{j} .
$$

Now, from the expression of $y_{1}\left(t_{1}\right)$ in $(2.3)$ we get

$$
y_{1}\left(t_{1}\right)=y\left(t_{1}\right)+B(t),
$$

where, if we put $q(t)=\varphi^{*}(q)$,

$$
B(t)=\frac{q(t) x^{\prime}(t)-p(t) y^{\prime}(t)}{x^{\prime}(t)}-A(t) .
$$

Proposition 5.1. Let $\varphi\left(t_{1}\right)=\left(t_{1}^{v_{0}}, y\left(t_{1}\right)\right)$, where $y\left(t_{1}\right)=t_{1}^{v_{1}}+t_{1}^{\lambda}+\sum_{i>\lambda} a_{i} t_{1}^{i}$, be a Puiseux parametrization, such that the genus of $\varphi$ is greater than 1 , and $n_{1}=2$. Then there exists $y_{1}\left(t_{1}\right)=t_{1}^{v_{1}}+t_{1}^{\lambda}+\sum_{i>\lambda} a_{i}^{\prime} t_{1}^{i}$, with $a_{i}^{\prime}=a_{i}$, for $i<v_{1}+\lambda-v_{0}$ and $a_{v_{1}+\lambda-v_{0}}^{\prime}=0$, such that $\left(t_{1}^{v_{0}}, y_{1}\left(t_{1}\right)\right) \sim_{\mathcal{A}}\left(t_{1}^{v_{0}}, y\left(t_{1}\right)\right)$.

Proof: Let $\beta \in \mathbb{C}$ and $p_{1}, q \in \mathcal{M}_{2}^{2}$, as above. We will show that we may choose $p_{1}$ and $q$, in such a way that $\operatorname{ord}_{t_{1}}(B(t))=v_{1}+\lambda-v_{0}$, where $B$ is as in (5.2), and then, by adjusting the value of $\beta$, we may make this term cancel the corresponding one in $y\left(t_{1}\right)$ in equation (5.1). 
Since $t_{1}=\rho(t)$, with $\rho$ an automorphism of $\mathcal{O}_{1}$, we have that

$$
\operatorname{ord}_{t_{1}}(B(t))=\operatorname{ord}_{t}(B(t))
$$

so, we may work with the powers of $t$ in the expression of $B(t)$.

Remark that $n_{1}=2$ implies that $m_{1} v_{0}=2 v_{1}$, where $m_{1}=\beta_{1} / e_{1}=v_{1} / e_{1}$.

Now we choose $p_{1}=0$ and $q=\frac{v_{1}}{v_{0}} \beta X^{m_{1}-1}+g$, with $g \in \mathcal{M}_{2}^{2}$ such that $v_{\varphi}(g)>\left(m_{1}-1\right) v_{0}$.

Let us write

$$
B(t)=B_{0}(t)+B_{1}(t)+B_{2}(t)
$$

where

$$
\begin{gathered}
B_{0}(t)=\beta \frac{\left(v_{1} / v_{0}\right) x(t)^{m_{1}-1} x^{\prime}(t)-y(t) y^{\prime}(t)}{x^{\prime}(t)}, \\
B_{1}(t)=\frac{g(t) x^{\prime}(t)}{x^{\prime}(t)}=g(t),
\end{gathered}
$$

and

$$
B_{2}(t)=-A(t)=-\sum_{i \geq v_{1}} a_{i} t^{i}\left(\sum_{j=2}^{\infty}\left(\begin{array}{c}
i / v_{0} \\
j
\end{array}\right)\left(\frac{\beta y(t)}{t^{v_{0}}}\right)^{j}\right) .
$$

A direct computation shows that, if $\beta \neq 0$, then

$$
\begin{gathered}
v_{\varphi}\left(B_{0}\right)=v_{\varphi}\left(\left(v_{1} / v_{0}\right) x(t)^{m_{1}-1} x^{\prime}(t)-y(t) y^{\prime}(t)\right)-\left(v_{0}-1\right)= \\
v_{1}+\lambda-1-v_{0}+1=v_{1}+\lambda-v_{0} .
\end{gathered}
$$

On the other hand, by expanding $B_{2}(t)$ one sees that there will be terms either of degree greater than $v_{1}+\lambda-v_{0}$, or of degree $r v_{0}+s v_{1}$, greater than $\left(m_{1}-1\right) v_{0}$, which can be eliminated by a suitable choice of $g(t)$.

With this last proposition we finished the proof of the existence part of Theorem 2.1, concerning the normal forms.

Now, to prove that if two Puiseux parametrizations are $\mathcal{A}$-equivalent, then they are conjugate under homothety, it will be sufficient to prove that if two Puiseux parametrizations are $\widetilde{\mathcal{A}}$-equivalent, then they are equal, because the $\mathcal{A}$-action is decomposed into the $\widetilde{\mathcal{A}}$-action and the $\mathcal{H}$-action.

Fixing a set $\Lambda$ of values of differentials in the equisingularity class determined by a semigroup $\Gamma$, let us consider the linear space

$$
N_{\Lambda}=\left\{\left(t^{v_{0}}, t^{v_{1}}+t^{\lambda}+\sum_{j>\lambda} a_{j} t^{j}\right) \in \Sigma_{\Gamma} ; a_{j}=0, \text { for } j \in \Lambda-v_{0}\right\} .
$$

If we denote by $N_{\Lambda}^{k}$ the space $j^{k}\left(N_{\Lambda}\right)$, we have the following lemma:

Lemma 5.2. If $\alpha \in N_{\Lambda}$, then for all $k>\lambda$, we have

$$
N_{\Lambda}^{k} \cap T_{j^{k}(\alpha)}\left(\widetilde{\mathcal{A}}^{k} \cdot j^{k}(\alpha)\right)=\left\{j^{k}(\alpha)\right\} .
$$


Proof: Suppose the assertion not true. Take $k$ minimal with the following property:

$$
N_{\Lambda}^{k} \cap T_{j^{k}(\alpha)}\left(\widetilde{\mathcal{A}}^{k} \cdot j^{k}(\alpha)\right) \neq\left\{j^{k}(\alpha)\right\} .
$$

So, there exists $\beta \in N_{\Lambda}^{k} \cap T_{j^{k}(\alpha)}\left(\widetilde{\mathcal{A}}^{k} \cdot j^{k}(\alpha)\right)$ such that $\beta \neq j^{k}(\alpha)$ and $j^{k-1}(\beta)=j^{k-1}(\alpha)$. Therefore, there exists $b \in \mathbb{C}^{*}$ such that

$$
\beta-j^{k}(\alpha)=\left(0, b t^{k}\right) \in T_{j^{k}(\alpha)}\left(\widetilde{\mathcal{A}}^{k} \cdot j^{k}(\alpha)\right) .
$$

Hence, from Proposition 3.3, it follows that $k \in \Lambda-v_{0}$. But, since $j^{k}(\alpha) \in N_{\Lambda}^{k}$, it follows that $j^{k}(\alpha)=j^{k-1}(\alpha)$. So, for some $b \neq 0$,

$$
\beta=j^{k-1}(\alpha)+\left(0, b t^{k}\right)
$$

But, since $\beta \in N_{\Lambda}^{k}$, one should have $b=0$, which is a contradiction.

Now we proceed to prove the uniqueness of the $\widetilde{\mathcal{A}}$-normal forms.

Let $\varphi(t)=\left(t^{v_{0}}, t^{v_{1}}+t^{\lambda}+\sum_{j>\lambda} a_{j} t^{j}\right) \in \Sigma_{\Gamma}$ be a Puiseux parametrization with $\Lambda_{\varphi}=\Lambda$. We denote by $\widetilde{\mathcal{A}}^{c-1} \cdot \varphi$ the orbit of $\varphi$ in $\Sigma_{\Gamma}$, with respect to the $\widetilde{\mathcal{A}}^{c-1}$-action.

We want to show that

$$
N_{\Lambda} \cap \widetilde{\mathcal{A}}^{c-1} \cdot \varphi=\{\varphi\} .
$$

Indeed, if $N_{\Lambda} \cap \widetilde{\mathcal{A}}^{c-1} \cdot \varphi \neq\{\varphi\}$, take $\varphi_{1} \in N_{\Lambda} \cap \widetilde{\mathcal{A}}^{c-1} \cdot \varphi$, with $\varphi_{1} \neq \varphi$. Since $\widetilde{\mathcal{A}}^{c-1} \cdot \varphi$ is arcwise connected, there exists an arc in $\widetilde{\mathcal{A}}^{c-1} \cdot \varphi$ joining $\varphi$ to $\varphi_{1}$. Since reduction to the normal form is continuous, it follows that $\varphi$ wouldn't be an isolated point in $N_{\Lambda} \cap \widetilde{\mathcal{A}}^{c-1} \cdot \varphi$. But this is a contradiction because of Lemma 5.2.

\section{Zariski's Problem and Computational Aspects}

Our methods, more than describing all normal forms (up to homotheties) of plane branches, with respect to analytic equivalence, give an effective way to obtain the normal form of a given branch and, as well, to distinguish from analytic point of view two given plane branches.

Indeed, since the set $\Lambda$ is an analytic invariant, it is the same for the branch and its normal form, so given two parametrizations $\varphi_{1}$ and $\varphi_{2}$ with same semigroup of values, we compute with the procedures exposed in [9] (specially Algorithm 4.10) the sets $\Lambda$ for both. If these are distinct, the branches are not equivalent. If they are equal, we proceed to put the parametrizations under they normal forms. To put a given parametrization $\varphi$ into its normal form, it is enough to consider changes of coordinates corresponding to an element of the group $\widetilde{\mathcal{A}}^{l-v_{0}}$, where $l$ is the greatest 
integer not in $\Lambda$. More precisely, taking

$$
p=\sum_{i} \alpha_{i} \prod_{j=0}^{g} h_{j}^{a_{i j}}, \quad q=\sum_{i} \gamma_{i} \prod_{j=0}^{g} h_{j}^{b_{i j}},
$$

where $h_{0}, h_{1}, \ldots, h_{g}$ are elements of a standard basis for the local ring $\mathcal{O}_{f}$ of the branch $(f)$ associated to $\varphi$ (see [9] for the definition (Definition 2.1) and how to compute them (Algorithm 3.2)), and the $\alpha_{i}$ 's and $\gamma_{i}$ 's are parameters, such that in the development in power series the smallest order term of $p$ is greater than $v_{0}$ and of $q$ is greater than $v_{1}$ and the terms of orders belonging to $\Gamma$ in $p$ (resp. $q$ ) are less than $l-v_{1}$ (resp. less than $l-v_{0}$ ).

Performing an action as (2.2) with the $p$ and $q$ as above, which is computationally possible, we impose conditions on the coefficients $\alpha_{i}$ and $\gamma_{i}$ in order to bring the parametrization into its normal form, in the way we did during the proof of Proposition 5.1.

This done, the analytic equivalence reduces to verify homothety, which is trivial.

Let us remark that Ebey (cf. [4, Theorem 5), by using arguments from the theory of algebraic groups, predicted the existence of some kind of normal forms under the $\mathcal{A}_{1}$-equivalence (cf. our Proposition 4.3) which he called canonical forms, but without any indication on how they could be obtained nor how they should look like.

The stratified moduli problem is also solved, since it is the disjoint union of quotients of a finite number of semi-algebraic sets, by finite groups, corresponding to the quotients modulo finitely many homotheties of the normal form corresponding to a given $\Lambda$ under the $\widetilde{\mathcal{A}}$-equivalence. The sets $\Lambda$ and the conditions on the coefficients that determine them, for a fixed equisingularity class, may be computed by the algorithms we developed in 9 . One of these set $\Lambda_{\text {gen }}$ corresponds to the generic branch, easily recognized by the open conditions on the coefficients.

Finally, the dimension of the component of the moduli corresponding to a given set $\Lambda$ is determined by the normal form and is at most equal to the number of gaps of $\Lambda$ greater that $\lambda$, since some of the coefficients of the parametrization may be fixed constants. The dimension of the generic component is exactly equal to the number of gaps of $\Lambda_{\text {gen }}$ greater that $\lambda$, since in this case, no coefficient in the corresponding normal form is constant.

\section{Some EXAMples}

In what follows we give two concrete examples of the application of our method. The first example will describe a result obtained in [8], and the second one is a new example which we relate to a question posed by Zariski in [11.

Example 7.1. The table below gives the analytic classification of plane branches in the equisingularity class of $\Gamma=\langle 6,9,19\rangle$. 


\begin{tabular}{|l|l|}
\hline Condition & Normal Form \\
\hline$b \notin\left\{-\frac{1}{2}, \frac{29}{18}\right\}$ & $\left(t^{6}, t^{9}+t^{10}+b t^{11}+b_{1} t^{14}+b_{2} t^{17}\right)$ \\
\hline$b=\frac{29}{18}$ & $\left(t^{6}, t^{9}+t^{10}+b t^{11}+b_{1} t^{14}+b_{2} t^{17}+b_{3} t^{23}\right)$ \\
\hline $\begin{array}{c}b=-\frac{1}{2} \\
A \neq 0\end{array}$ & $\left(t^{6}, t^{9}+t^{10}+b t^{11}+b_{1} t^{14}+b_{2} t^{17}+b_{3} t^{20}\right)$ \\
\hline $\begin{array}{c}b=-\frac{1}{2} \\
A=0\end{array}$ & $\left(t^{6}, t^{9}+t^{10}+b t^{11}+b_{1} t^{14}+b_{2} t^{17}+b_{3} t^{20}+b_{4} t^{26}\right)$ \\
\hline
\end{tabular}

Where $A=14+\frac{769}{2} b_{1}-532 b_{2}-576 b_{1}^{2}$. Moreover, two branches belonging to the same normal form are equivalent if, and only if, they are equal.

In the above example we have that the stratified moduli space consists of four strata, all of them of dimension 3 , with the first one corresponding to the generic stratum.

Example 7.2. Our second example deals with the classification of the equisingularity class given by the semigroup of values $\Gamma=\langle 7,8\rangle$.

Since the conductor of $\Gamma$ is 42 , we have

$$
\Sigma_{\Gamma}=\left\{\left(t^{7}, t^{8}+\sum_{8<i<42} a_{i} t^{i}\right) ; a_{i} \in \mathbb{C}\right\} .
$$

Algorithm 4.10 of [9] and Theorem 2.1 give the following table:

\begin{tabular}{|c|c|c|}
\hline Condition & Normal Form & $\Lambda \backslash \Gamma$ \\
\hline$a_{12} \neq \frac{13+9 a_{11}^{2}}{8}$ & $\left(t^{7}, t^{8}+t^{10}+a_{11} t^{11}+a_{12} t^{12}+a_{13} t^{13}+a_{20} t^{20}\right)$ & $\begin{array}{l}17,25,26 \\
33,34,41\end{array}$ \\
\hline$a_{13} \neq \frac{39}{10} a_{11}+\frac{27}{20} a_{11}^{3}$ & $\left(t^{7}, t^{8}+t^{10}+a_{11} t^{11}+\frac{13+9 a_{11}^{2}}{8} t^{12}+a_{13} t^{13}+a_{19} t^{19}\right)$ & $\begin{array}{l}17,25,27 \\
33,34,41\end{array}$ \\
\hline$a_{20} \neq B$ & $\begin{aligned}\left(t^{7}, t^{8}+t^{10}+\right. & a_{11} t^{11}+\frac{13+9 a_{11}^{2}}{8^{8}} t^{12}+ \\
& \left.\left(\frac{39}{10} a_{11}+\frac{27}{20} a_{11}^{3}\right) t^{13}+a_{19} t^{19}+a_{20} t^{20}\right)\end{aligned}$ & $\begin{array}{c}17,25,33 \\
34,41 \\
\end{array}$ \\
\hline \multirow[t]{3}{*}{$a_{20}=B$} & $\begin{array}{l}\left(t^{7}, t^{8}+t^{10}+a_{11} t^{11}+\frac{13+9 a_{11}^{2}}{8} t^{12}+\right. \\
\left.\quad+\left(\frac{39}{10} a_{11}+\frac{27}{20} a_{11}^{3}\right) t^{13}+a_{19} t^{19}+a_{20} t^{20}+a_{27} t^{27}\right)\end{array}$ & $\begin{array}{l}17,25 \\
33,41 \\
\end{array}$ \\
\hline & $\left(t^{7}, t^{8}+t^{11}+a_{12} t^{12}+a_{13} t^{13}+a_{20} t^{20}\right)$ & $\begin{array}{l}18,25,26 \\
33,34,41\end{array}$ \\
\hline & $\left(t^{7}, t^{8}+t^{12}+a_{13} t^{13}+a_{18} t^{18}\right)$ & $\begin{array}{l}19,26,27 \\
33,34,41\end{array}$ \\
\hline \multirow[t]{2}{*}{$a_{18} \neq-\frac{1}{2}$} & $\left(t^{7}, t^{8}+t^{13}+a_{18} t^{18}+a_{19} t^{19}+a_{26} t^{26}\right)$ & $\begin{array}{c}20,27,33 \\
34,41 \\
\end{array}$ \\
\hline & $\left(t^{7}, t^{8}+t^{13}-\frac{1}{2} t^{18}+a_{19} t^{19}+a_{26} t^{26}\right)$ & $\begin{array}{l}20,27 \\
34,41\end{array}$ \\
\hline \multirow[t]{8}{*}{$a_{20} \neq \frac{121}{120} a_{19}^{2}$} & $\left(t^{7}, t^{8}+t^{18}+a_{19} t^{19}+a_{20} t^{20}+a_{27} t^{27}\right)$ & $\begin{array}{l}25,33 \\
34,41\end{array}$ \\
\hline & $\left(t^{7}, t^{8}+t^{18}+a_{19} t^{19}+\frac{121}{120} a_{19}^{2} t^{20}+a_{27} t^{27}\right)$ & $25,33,41$ \\
\hline & $\left(t^{7}, t^{8}+t^{19}+a_{20} t^{20}\right)$ & $\begin{array}{l}26,33 \\
34,41\end{array}$ \\
\hline & $\left(t^{7}, t^{8}+t^{20}+a_{26} t^{26}\right)$ & $27,34,41$ \\
\hline & $\left(t^{7}, t^{8}+t^{26}+a_{27} t^{27}\right)$ & 33,41 \\
\hline & $\left(t^{7}, t^{8}+t^{27}\right)$ & 34,41 \\
\hline & $\left(t^{7}, t^{8}+t^{34}\right)$ & 41 \\
\hline & $\left(t^{7}, t^{8}\right)$ & $\emptyset$ \\
\hline
\end{tabular}


where

$$
B=\frac{11}{4} a_{11} a_{19}-\frac{357}{512}-\frac{47399}{2560} a_{11}^{2}-\frac{10097}{320} a_{11}^{4}-\frac{17523}{1280} a_{11}^{6}-\frac{2187}{1280} a_{11}^{8} .
$$

Two parametrizations of the above table on the same line are equivalent if and only if they are homothetic, with respect to the appropriate root of unity $((\lambda-8)$-th root of unity).

From the above table we see that the generic component of the moduli, corresponding to parametrizations on the first line, has dimension 4 . There are six strata of dimension 3 , three strata of dimension 2 , three strata of dimension 1 and three strata of dimension 0 .

Zariski dedicated Sections 4,5 and 6 of Chapter VI, in [11], to the study of branches with semigroups of the form $\Gamma=\left\langle v_{0}, v_{0}+1\right\rangle$, where the following result is proved:

TheOREM ([11], Théorème 6.12) Let $v_{0} \geq 5$, and for all $s \in\left\{2, \ldots, v_{0}-2\right\}$, define

$$
\mathcal{L}_{s}=\left\{s v_{0}+s+2, s v_{0}+s+3, \ldots, s v_{0}+s+v_{0}-1-s\right\}
$$

Let

$$
\varphi=\left(t^{v_{0}}, t^{v_{0}+1}+a_{v_{0}+3} t^{v_{0}+3}+\cdots+a_{2 v_{0}-1} t^{2 v_{0}-1}+\sum_{i \in \cup_{s=2}^{q} \mathcal{L}_{s}} a_{i} t^{i}\right),
$$

where $q=\left[\frac{v_{0}-3}{2}\right]$ and $a_{i}=0$, whenever $i$ is one of the first $s+1$ elements of $\mathcal{L}_{s}$, for all $2 \leq s \leq q$.

Then two generic parametrizations of the above form are $\mathcal{A}$-equivalent if, and only if, they homothetic.

In [11, Zariski remarks that the above theorem is true for $2 \leq v_{0} \leq 6$ without the condition on the genericity of the parameters (Remarque 6.14), and asks the following question:

Is the above theorem true without the assumption of the genericity on the coefficients of the parametrizations?

The answer is no! And an example may be given considering branches with semigroup $\Gamma=\langle 7,8\rangle$.

Consider

$$
\varphi=\left(t^{7}, t^{8}+t^{10}+t^{11}+\frac{11}{4} t^{12}+a_{13} t^{13}+a_{20} t^{20}\right)
$$

with $a_{13} \neq \frac{21}{4}$. Obviously, $\varphi$ is in the form of the above theorem.

If we consider changes of coordinates as in (2.2) with

$$
p=b_{1} x^{2}-\frac{3}{2} b_{1} x y-\frac{1}{4} b_{1} y^{2}+b_{2} x^{3}+b_{3} x^{2} y+b_{4} x y^{2}+b_{5} y^{3}
$$

and

$$
q=\frac{8}{7} b_{1} x y-\frac{12}{7} b_{1} y^{2}+\left(-\frac{135}{28} b_{1}-\frac{15}{14} a_{13} b_{1}\right) x^{3}+b_{6} x^{2} y+b_{7} x y^{2}+b_{8} y^{3}
$$


where

$$
\begin{aligned}
b_{2}= & \frac{4}{7} b_{1}^{2}-\frac{227}{6} b_{1}+\frac{199}{24} a_{13} b_{1}-\frac{2}{3} b_{3}-\frac{8}{3} b_{5}, \\
b_{3}= & 6 b_{4}-\frac{45}{2} b_{1} a_{13}^{2}+\frac{9565}{16} b_{1}-40 b_{8}+\frac{72}{7} b_{1}^{2}+\frac{4297}{16} a_{13} b_{1}, \\
b_{4}= & \left(4 a_{13}-21\right)^{-1} \frac{1}{1120}\left(-2720 a_{13} b_{1}^{2}+557690 a_{13} b_{1}-\right. \\
& 277200 b_{1} a_{13}^{2}+16800 b_{1} a_{13}^{3}+26880 a_{13} b_{5}+ \\
& \left.2459289 b_{1}-141120 b_{5}+14280 b_{1}^{2}+2688 b_{1} a_{20}\right), \\
b_{6}= & -\frac{25}{4} b_{1}-\frac{29}{28} a_{13} b_{1}+\frac{8}{7} b_{2}+\frac{4}{49} b_{1}^{2} \\
b_{7}= & -\frac{3}{2} a_{13} b_{1}+\frac{8}{7} b_{3}-\frac{641}{56} b_{1}-\frac{12}{49} b_{1}^{2} \\
b_{8}= & -\frac{52}{7} b_{1}+\frac{8}{7} b_{4}-\frac{81}{28} a_{13} b_{1}+\frac{2}{7} b_{2}+\frac{18}{49} b_{1}^{2},
\end{aligned}
$$

we have

$\sigma \circ \varphi \circ \rho^{-1}\left(t_{1}\right)=\left(t_{1}^{7}, t_{1}^{8}+t_{1}^{10}+t_{1}^{11}+\frac{11}{4} t_{1}^{12}+a_{13} t_{1}^{13}+\left(a_{20}+5 b_{1}\left(\frac{3}{4}-\frac{1}{7} a_{13}\right)\right) t_{1}^{20}\right)$.

By choosing conveniently $b_{1}$ we see that the normal form of $\varphi$ is given in the second row of the table in Example 7.2, but $\varphi$ itself is not in normal form (this gives the reduction of $\varphi$ to normal form).

Since $a_{13} \neq \frac{21}{4}$, then for each $b_{1} \neq 0$ we get a parametrization $\mathcal{A}$-equivalent to $\varphi$, as described in Zariski's Theorem, without being homothetically equivalent to $\varphi$, giving a negative answer to Zariski's question.

\section{REFERENCES}

[1] Bruce, J. W. and Gaffney, T. J. - Simple Singularities of Mappings $\mathbb{C}, 0 \longrightarrow \mathbb{C}^{2}, 0$. J. London Math. Soc. (2), 26, 465-474 (1982)

[2] Bruce, J. W., Kirk, N. P. and du Plessis, A. A. - Complete Transversals and the Classification of Singularities. Nonlinearity, 10, N. 1, 253-275 (1997)

[3] Delorme, C. - Sur les Modules des Singularités de Courbes Planes. Bull. Soc. Math. France 106, 417-446 (1978)

[4] Ebey, S. - The Classification of Singular Points of Algebraic Curves. Trans. Amer. Math. Soc. 118, 454-471 (1965)

[5] Gibson, C. G. - Singular points of smooth mappings, Research Notes in Math. 25, Pitman, London, 1973.

[6] Hefez, A. - Irreducible Plane Curve Singularities. In Real and Complex Singularities, D. Mond and M. J. Saia, Editors, Lecture Notes in Pure and Applied Math. Vol. 232, Marcel Dekker, 1-120 (2003)

[7] Hefez, A. and Hernandes, M. E. - Computational Methods in the Local Theory of Curves. $23^{\circ}$ Colóquio Brasileiro de Matemática, Publicações Matemáticas, IMPA (2001)

[8] Hefez, A. and Hernandes, M. E. - Classification of Algebroid Plane Curves with Semigroup $\langle 6,9,19\rangle$. Comm. Algebra 31 (8), 3847-3861, (2003)

[9] Hefez, A. and Hernandes, M. E. - Standard bases for local rings of branches and their modules of differentials. J. Symb. Comput. 42, 178-191, (2007)

[10] Robbiano, L. and Sweedler, M. - Subalgebra Bases. Proc. Commutative Algebra, Salvador, SLN in Math. 1430, 61-87, Springer-Verlag (1988)

[11] Zariski, O. - Le Problème des Modules pour les Branches Planes. Cours donné au Centre de Mathématiques de L'École Polytechnique. Nouvelle éd. revue par l'auteur. Rédigé par François Kimety et Michel Merle. Avec un appendice de Bernard Teissier. Paris, Hermann (1986).

English translation by Ben Lichtin: The Moduli Problem for Plane Branches. University Lecture Series, Vol. 39, AMS (2006) 
[12] Zariski, O. - Characterization of Plane Algebroid Curves whose Module of Differentials has Maximum Torsion. Proc. Nat. Acad. of Sc. U.S.A., 56, 781-786 (1966)

\author{
Abramo Hefez \\ Universidade Federal Fluminense \\ Instituto de Matemática \\ R. Mario Santos Braga, s/n \\ 24020-140 Niterói, RJ - Brazil \\ E-mail: hefez@mat.uff.br \\ Marcelo E. Hernandes \\ Universidade Estadual de Maringá \\ Departamento de Matemática \\ Av. Colombo, 5790 \\ 87020-020 Maringá, PR - Brazil \\ E-mail: mehernandes@uem.br
}

